\title{
Conditions de formation de composés organoiodés sapides lors de l'oxydation par le chlore d'eaux contenant des ions iodure Conditions of formation of organoiodinated compounds during chlorination of aqueous solutions containing iodide ions
}

\author{
N. Karpel Vel Leitner, J. Vessela, M. Doré, J. P. Gautier et E. Lefebvre
}

Volume 11, numéro 3, 1998

URI : https://id.erudit.org/iderudit/705316ar

DOI : https://doi.org/10.7202/705316ar

Aller au sommaire du numéro

Éditeur(s)

Université du Québec - INRS-Eau, Terre et Environnement (INRS-ETE)

ISSN

0992-7158 (imprimé)

1718-8598 (numérique)

Découvrir la revue

Citer cet article

Karpel Vel Leitner, N., Vessela, J., Doré, M., Gautier, J. P. \& Lefebvre, E. (1998). Conditions de formation de composés organoiodés sapides lors de l'oxydation par le chlore d'eaux contenant des ions iodure. Revue des sciences de l'eau / Journal of Water Science, 11(3), 445-457. https://doi.org/10.7202/705316ar

\section{Résumé de l'article}

Le travail a consisté à préciser les conditions de formation d'une molécule iodée sapide, l'iodoforme, lors de l'oxydation d'une eau brute par le chlore et à proposer une voie réactionnelle possible.

L'étude de la chloration d'une eau brute en présence d'azote ammoniacal et d'ions iodure conduit à la formation d'iodoforme uniquement pour des taux inférieurs au point de rupture. Les résultats montrent que l'oxydation de l'ion ammonium conduit à la formation de monochloramine dont le pouvoir oxydant totalement disponible pourrait être impliqué dans la formation de iodamines ou de chloroiodamines. Ces réactions sont plus favorables en présence d'iode qu'en présence d'ions iodure. Mais l'action de l'iode seul en présence d'ammoniaque et en absence de monochloramine ne permet pas d'expliquer la production des composés organoiodés observés. Ce sont les précurseurs intermédiaires formés à partir des chloramines qui, par action sur la matière organique naturelle, seraient responsables de la formation d'iodoforme. Dans une moindre mesure, certains composés azotés organiques tels les amines et les acides aminés pourraient prendre part à la production des composés organoiodés lors de la chloration.
Tous droits réservés @ C Revue des sciences de l'eau, 1998

Ce document est protégé par la loi sur le droit d'auteur. L'utilisation des services d'Érudit (y compris la reproduction) est assujettie à sa politique d'utilisation que vous pouvez consulter en ligne.

https://apropos.erudit.org/fr/usagers/politique-dutilisation/ 


\title{
Conditions de formation de composés organoiodés sapides lors de l'oxydation par le chlore d'eaux contenant des ions iodure
}

\author{
Conditions of formation of organoiodinated compounds \\ during chlorination of aqueous solutions containing \\ iodide ions
}

\section{N. KARPEL VEL LEITNER ${ }^{1 *}$, J. VESSELLA ${ }^{1}$, M. DORE 1 , J.P. GAUTIER ${ }^{2}$ et E. LEFEBVRE ${ }^{3}$}

Reçu le 12 mai 1997, accepté le 23 février 1998**.

\section{SUMMARY}

This work consisted in specifying the conditions of iodoform formation during chlorination of a raw water containing iodides. To reach this objective, there was need to spike the studied natural water with potassium iodide $\left(200 \mu \mathrm{g} \cdot \mathrm{L}^{-1}\right)$ in order to increase the low natural iodide content. Free and combined chlorine, chlorinated and brominated trihalomethanes (THMs) and iodoform were analyzed.

It was shown that:

- iodoform is formed for chlorine doses prior to the breakpoint, in a region where the formation of the most classical chlorinated and brominated THMs is usually disfavored (figure 1-4);

- in the presence of chloramines the rate of production of iodoform increases with increasing $\mathrm{I}^{-}$or $\mathrm{I}_{2}$ (figure 5);

- the direct reaction of $I_{2}$ with THMs precursors to produce iodoform is slow and independent of the presence of ammonia (table 1);

- nitrogenated compounds such as amines and aminoacids would also take part in the production of organoiodinated compounds during chlorination (figure 7). However, under water treatment conditions, taking into account the amine and amino acids content of natural waters, this class of compounds will only take a small part in the mechanism of iodoform formation.

Among the possible routes that could account for the observations made in this research, the formation of iodamines or chloroiodamines as intermediates is suggested (figure 8). From a practical point of view, the removal of ammonia from water by a biological process (nitrification step) would inhibit the iodo-

1. Laboratoire de Chimie de l'Eau et de l'Emvironnement, ESIP, UPRES A 6008, 40 avenue du Recteur Pineau, 86022 Poitiers cedex.

2. ELF-ATOCHEM, Centre d'Application de Levallois, 95 rue Danton, 92300 Levallois-Perret.

3. SAUR, Centre Pierre Crussard, 78312 Maurepas cedex.

* Correspondance.

** Les commentaires seront reçus jusqu'au 30 avril 1999. 
form formation potential and allow the application of the final chlorination step. Another alternative would involve replacing the chlorination step by oxidation with chlorine dioxide.

Key-words: chlorine, iodide, chloramine, iodoform.

\section{RÉSUMÉ}

Le travail a consisté à préciser les conditions de formation d'une molécule iodée sapide, l'iodoforme, lors de l'oxydation d'une eau brute par le chlore et à proposer une voie réactionnelle possible.

L'étude de la chloration d'une eau brute en présence d'azote ammoniacal et d'ions iodure conduit à la formation d'iodoforme uniquement pour des taux inférieurs au point de rupture. Les résultats montrent que l'oxydation de l'ion ammonium conduit à la formation de monochloramine dont le pouvoir oxydant totalement disponible pourrait être impliqué dans la formation de iodamines ou de chloroiodamines. Ces réactions sont plus favorables en présence d'iode qu'en présence d'ions iodure. Mais l'action de l'iode seul en présence d'ammoniaque et en absence de monochloramine ne permet pas d'expliquer la production des composés organoiodés observés. Ce sont les précurseurs intermédiaires formés à partir des chloramines qui, par action sur la matière organique naturelle, seraient responsables de la formation d'iodoforme. Dans une moindre mesure, certains composés azotés organiques tels les amines et les acides aminés pourraient prendre part à la production des composés organoiodés lors de la chloration.

Mots clés : chlore, iodure, chloramine, iodoforme.

\section{1 - INTRODUCTION}

Les propriétés oxydantes du chlore sont encore largement utilisées dans les filières d'eau potable où ce réactif est introduit en désinfection finale mais également en préoxydation. Outre la formation maintenant bien connue de trihalométhanes (THM), diverses études mentionnent depuis quelques années des problèmes de goûts et d'odeurs qui seraient liés à l'utilisation de cet oxydant (MONTIEL et al., 1987 ; BRUCHET et al., 1989). Les THM bromés, chlorés et iodés formés lors de la chloration d'eaux contenant des traces d'ions bromure ou iodure seraient à l'origine de goût et d'odeur pharmaceutiques. La concentration seuil de goût pour l'iodoforme dont la toxicité a été peu étudiée à ce jour (ROLDAN-ARJONA et al., 1991 ; ROLDAN-ARJONA and PUEYO, 1993) est de l'ordre de $5 \mu \mathrm{g} / \mathrm{L}$ (HANSSON et al, 1987). Associé à la présence d'autres THM iodés (THOMAS et al., 1980), ce composé permettrait d'expliquer les problèmes rencontrés dans de nombreuses eaux contenant des ions iodure généralement présents dans les eaux souterraines à une concentration de 0,01 à $20 \mu \mathrm{g} \mathrm{I} / \mathrm{L}$ (ALI-MOHAMED and JAMALI, 1989). La chloramination de l'eau favoriserait toutefois la formation de composés organoiodés par rapport à la chloration (BRUCHET et al., 1989). HANSSON et al. (1987) ont ainsi montré que la production d'iodoforme dépend de l'ordre d'introduction du chlore et de l'ammoniaque. GITTELMAN et YOLE (1989) observent également que la succession chlore/ammoniaque diminue d'autant 
plus les odeurs pharmaceutiques par rapport à la chloramination (succession $\mathrm{NH}_{3} / \mathrm{Cl}_{2}$ ) que le temps de réaction avec le chlore, avant l'addition d'ammoniaque, est élevé.

Nos travaux entrent dans le cadre de l'étude de l'action de différents oxydants sur des eaux contenant des halogénures et concernent en particulier la détermination de mécanismes permettant d'expliquer la formation de composés organohalogénés. Les expériences réalisées au laboratoire à partir d'une eau brute dopée en ions iodure ont permis de définir la nature de l'entité responsable de la formation d'iodoforme lors de l'oxydation par le chlore.

\section{2 - MATÉRIELS ET MÉTHODES}

\subsection{Origine des solutions et traitements d'oxydation}

Les expériences ont été réalisées à partir d'une eau (notée EB1) issue d'une nappe alluviale ([Carbone Organique Total $]=2,5 \mathrm{mg} \mathrm{C} / \mathrm{L} ; \mathrm{pH}=8,0$ ). Pendant la durée de l'étude (période de prélèvements: janvier 1995-juin 1996), la teneur naturelle en ions iodure a toujours été comprise entre 20 et $30 \mu \mathrm{g} / \mathrm{L}$. L'eau étudiée est préalablement oxygénée afin d'oxyder les ions ferreux en ions ferrique. Pour les expériences réalisées en présence d'ions ammonium, l'eau brute aérée a ensuite été filtrée sur une membrane de porosité $1,2 \mu \mathrm{m}$ afin d'éliminer les matières en suspension. La concentration en ions ammonium a généralement été de l'ordre de $0,8 \pm 0,1 \mathrm{mg} \mathrm{N}-\mathrm{NH}_{4}{ }^{+} / \mathrm{L}$. Pour les travaux réalisés en absence d'ions ammonium, l'eau brute après aération a été filtrée sur une colonne de sable ensemencé de bactéries nitrifiantes permettant une nitrification complète (transformation de la totalité des ions ammonium en ions nitrate). Compte tenu des limites de détection offertes par les méthodes analytiques, pour la plupart des expériences les solutions ont été dopées en ions iodure à une concentration de $200 \mu \mathrm{g} / \mathrm{L}$. Ces teneurs, bien qu'importantes, peuvent être rencontrées dans certaines eaux de forage.

Toutes les expériences ont été menées à température ambiante $\left(20 \pm 2^{\circ} \mathrm{C}\right)$ dans des flacons ambrés de 1 litre à bouchon rodé.

Les taux d'oxydant appliqués ont généralement été de $1,5 \mathrm{mg} \mathrm{Cl} / \mathrm{L}$. La chloration de l'eau brute est réalisée par ajout d'un faible volume d'une solution fille de chlore de concentration $1 \mathrm{~g} / \mathrm{L}$ préparée à partir d'une solution commerciale d'hypochlorite de sodium et amenée à $\mathrm{pH} 8$. La réduction du chlore total résiduel, généralement effectuée après $6 \mathrm{~h}$ de temps de contact, a été obtenue par ajout de métaarsenite de sodium. L'action de cet agent déchlorant qui, utilisé dans un rapport stœchiométrique de 1 , permet une réduction rapide du chlore, s'est avérée négligeable vis-à-vis des composés organohalogénés analysés.

Pour les expériences de chloramination, une solution mère de monochloramine (400 mg/L) a été préparée dans de l'eau ultra-pure tamponnée par des ions phosphate $(\mathrm{pH}=8,5)$ en présence d'ammoniaque, par introduction d'ions hypochlorite dans un rapport molaire chlore/ammoniaque $=0,8$ dans le but de limiter la teneur en chlore libre résiduel $(<3 \%)$. À l'issue des réactions de chloramination, les chloramines résiduelles sont neutralisées par ajout d'ions sulfite. Aucune 
réactivité significative de ce réducteur vis-à-vis de l'iodoforme et des THM chlorés et bromés n'a été observée pour les concentrations mesurées au cours de notre étude.

\subsection{Méthodes analytiques}

Les différentes formes du chlore (chlore libre, chloramines, chlore total) ont été dosées par la méthode colorimétrique à la $\mathrm{N}, \mathrm{N}$-DiéthylPhénylène-1,4 Diamine (DPD) en réduisant d'un facteur 20 les volumes de réactifs et de prélèvements par rapport au protocole du Standard Methods (1995) selon les indications de VENTRESQUE et al. (1990). Cette méthode permet de mesurer des concentrations en chlore dans la gamme de 0,05 à $2,0 \mathrm{mg} \mathrm{Cl}_{2} \mathrm{~L}$.

Le dosage des ions iodure a été réalisé par potentiométrie à l'aide d'une électrode spécifique des ions iodure (Radiometer XS230) et d'une électrode de référence (XR 200) en présence de nitrate de sodium comme électrolyte $\left(10^{-2} \mathrm{~mol} / \mathrm{L}\right)$. La limite de détection ainsi obtenue est de $10 \mu \mathrm{g} \mathrm{I}^{-} / \mathrm{L}$.

L'analyse d'iodoforme mise au point dans notre laboratoire est effectuée en présence d'un étalon interne, le 1,1,1,2-tétrachloroéthane, par chromatographie en phase gazeuse après extraction liquide-liquide au pentane. La séparation est réalisée sur une colonne capillaire DB1701 à partir d'une programmation de température de $50^{\circ} \mathrm{C}$ à $100^{\circ} \mathrm{C}\left(10^{\circ} \mathrm{C} / \mathrm{min}\right)$ suivie d'une montée jusqu'à $180^{\circ} \mathrm{C}$ (à $5^{\circ} \mathrm{C} / \mathrm{min}$ ). La limite de détection obtenue dans ces conditions avec un détecteur à capture d'électrons est de $0,3 \mu \mathrm{g} / \mathrm{L}$ pour le iodoforme.

Les THM chlorés et/ou bromés ont été dosés par chromatographie en phase gazeuse à partir d'un système d'injection automatique par espace de tête (DANI HSS 3950 ; température du bain : $40^{\circ} \mathrm{C}$ ) sur une colonne capillaire DB 624 . La programmation de température a été de $50^{\circ} \mathrm{C}$ à $120^{\circ} \mathrm{C}\left(5^{\circ} \mathrm{C} / \mathrm{min}\right)$ et le détecteur à capture d'électrons utilisé a permis d'atteindre une limite de détection dans ces conditions de $0,5 \mu \mathrm{g} / \mathrm{L}$ de chloroforme.

\section{3 - RÉSULTATS}

\section{Rôle des ions ammonium}

L'eau brute EB1, dopée en ions iodure $\left(\left[\mathrm{I}^{-}\right]=260 \mu \mathrm{g} / \mathrm{L}\right)$, a été traitée par différentes doses de chlore (2 à $12 \mathrm{mg} / \mathrm{L}$ ) permettant d'obtenir la valeur du point de rupture (avec $\left[\mathrm{N}-\mathrm{NH}_{4}{ }^{+}\right]_{0}=0,69 \mathrm{mg} / \mathrm{L}$ ). La figure 1 présente l'évolution de la teneur en chlore libre, chlore total et chlore combiné après un temps de contact de $6 \mathrm{~h}$.

Pour différents taux de chloration inférieurs et supérieurs au point de rupture $\left(8,7 \mathrm{mg} \mathrm{Cl}_{2} / \mathrm{mg} \mathrm{N}-\mathrm{NH}_{4}{ }^{+}\right)$les mesures de trihalométhanes (THM) chlorés, bromés et iodés présentées dans la figure 2 indiquent :

- La teneur en THM chlorés et bromés augmente avec le taux de chloration. La production de dibromochlorométhane n'apparaît qu'au-delà du point de rupture et le bromoforme est observé uniquement pour le taux de chloration le plus

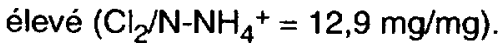


- En deçà du point de rupture, la compétition entre les différentes espèces présentes $\left(\mathrm{NH}_{3}, \mathrm{I}^{-}, \mathrm{Br}\right.$, précurseurs...) vis-à-vis de l'acide hypochloreux privilégie la formation de chloramines et contribue à limiter la production des THM classiques. Pour ces taux, on observe néanmoins la formation d'iodoforme dont la concentration (9 à $14 \mu \mathrm{g} / \mathrm{L}$ ) n'évolue que très peu avec la dose d'oxydant. Pour les taux de chloration supérieurs au point de rupture on n'observe pas de formation de $\mathrm{CHI}_{3}$.

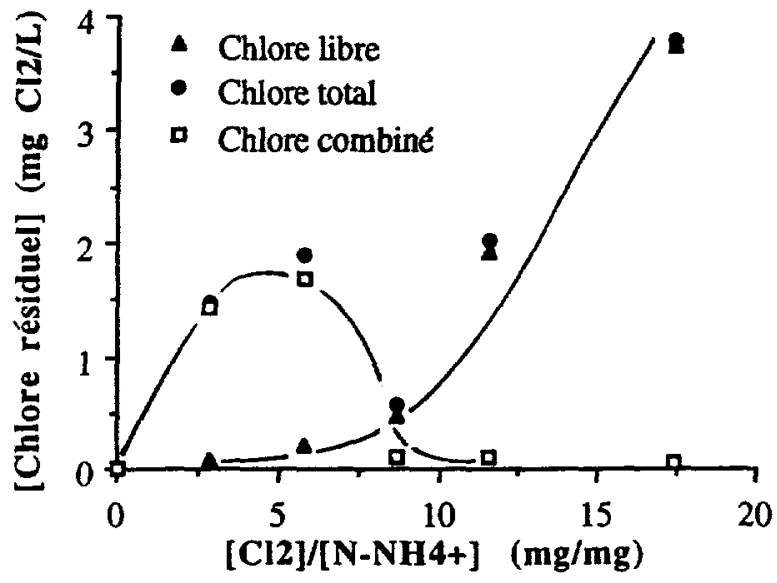

Figure 1 Chloration de l'eau brute EB1: Influence du rapport $\left[\mathrm{Cl}_{2}\right] /\left[\mathrm{N}-\mathrm{NH}_{4}{ }^{+}\right]$. [N$\left.\mathrm{NH}_{4}{ }^{+}\right]_{0}=0,69 \mathrm{mg} / \mathrm{L} ;\left[I^{-}\right] \mathrm{o}=260 \mu \mathrm{g} / \mathrm{L}$.

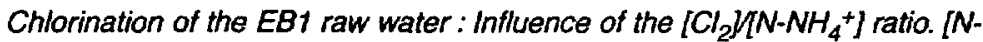
$\left.\mathrm{NH}_{4}\right]_{o}=0.69 \mathrm{mg} / \mathrm{L} ;[\mathrm{r} \mathrm{Jo}=260 \mu \mathrm{g} L$.

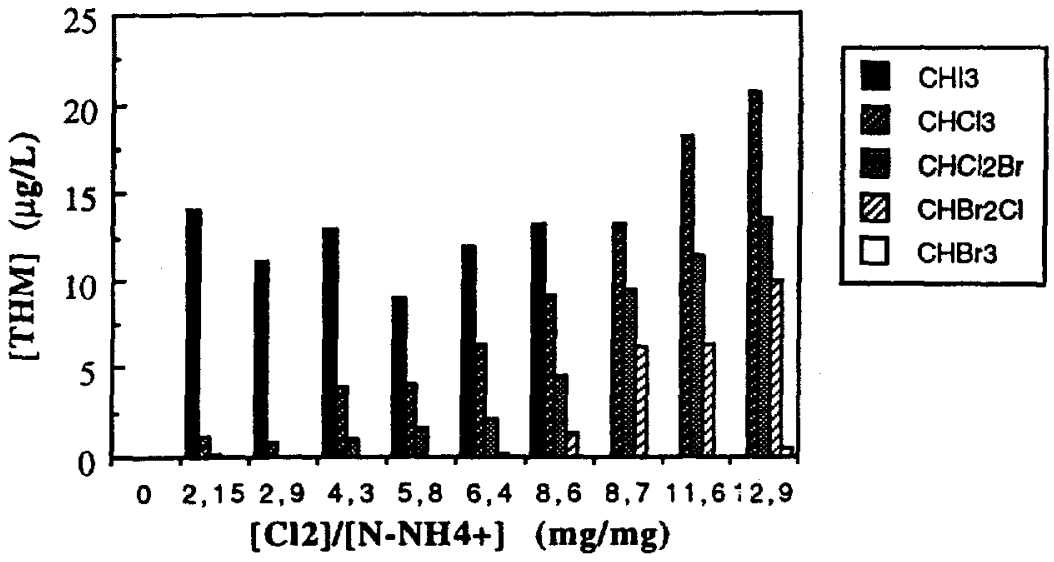

Figure 2 Chloration de l'eau brute EB1 : Influence du taux de chloration sur la formation des THM. $\left[\mathrm{N}-\mathrm{NH}_{4}{ }^{+}\right]_{0}=0,69 \mathrm{mg} / \mathrm{L} ;\left[\mathrm{I}^{-}\right]_{0}=260 \mu \mathrm{g} / \mathrm{L}$.

Chlorination of the EB1 raw water : Influence of the chlorine dose on THMS

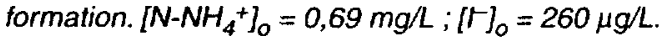


Des expériences comparables ont été réalisées en appliquant un taux de chloration fixe (10 $\mathrm{mg} / \mathrm{L}$ ) à l'eau brute EB1 dopée en ions iodure et additionnée de différentes teneurs en azote ammoniacal $\left(0,05\right.$ à $\left.6 \mathrm{mg} \mathrm{NH}_{4}{ }^{+} / \mathrm{L}\right)$ après nitrification biologique (figure 3 ).

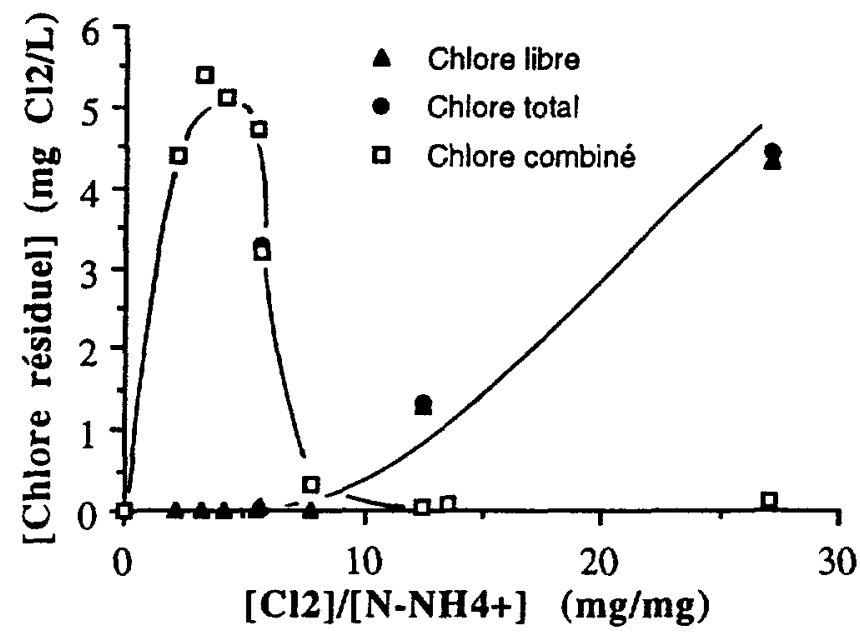

Figure 3 Chloration de l'eau brute EB1 : Influence de la concentration en ions ammonium. $\left[\mathrm{Cl}_{2}\right]_{0}=10 \mathrm{mg} / \mathrm{L} ;\left[\mathrm{NH}_{4}{ }^{+}\right]_{0}=0,05$ à $6 \mathrm{mg} \mathrm{NH}_{4}{ }^{+} / \mathrm{L} ;\left[\mathrm{I}^{-}\right]_{0}=200 \mu \mathrm{g} / \mathrm{L}$; $\mathrm{pH}_{\mathrm{o}}=7,9$.

Chlorination of the EB1 raw water: Influence of the ammonia concentration.

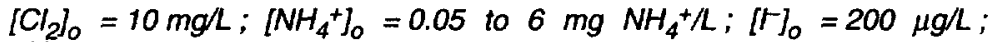
$\mathrm{pH}_{0}=7.9$.

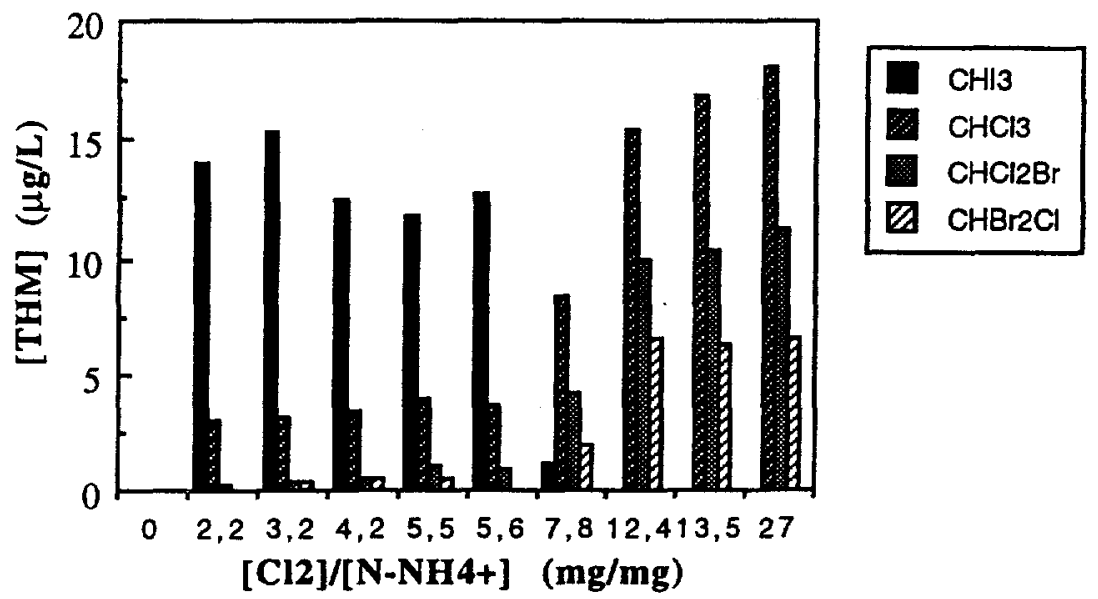

Figure 4 Chloration de l'eau brute EB1: Influence de la teneur en ammoniaque sur la formation des THM (conditions identiques à la figure 3).

Chlorination of the EBI raw water : Influence of the ammonia concentration on THMS formation. 
Comme pour l'expérience précédente sur l'étude de l'influence du taux de chloration, on constate après un temps de contact de $6 \mathrm{~h}$ :

- des concentrations en THM chlorés et bromés faibles avant le point de rupture $\left(\mathrm{Cl}_{2} / \mathrm{N}^{-\mathrm{NH}_{4}}{ }^{+}<7,8 \mathrm{mg} / \mathrm{mg} ; \mathrm{CHCl}_{3}<5 \mu \mathrm{g} / \mathrm{L}\right)$ et beaucoup plus importantes au-delà (figure 4);

- une formation d'iodoforme relativement constante en deçà du point de rupture $(\sim 12 \mu \mathrm{g} / \mathrm{L})$ et nulle au-delà. La comparaison des figures 2 et 4 indique que pour des rapports $\mathrm{Cl}_{2} / \mathrm{N}-\mathrm{NH}_{4}{ }^{+}$compris entre 2 et 6,5 , la production d'iodoforme est indépendante de la concentration en ions ammonium ( $\left[\mathrm{N}-\mathrm{NH}_{4}{ }^{+}\right]=0,7$ à $6 \mathrm{mg} / \mathrm{L}$ ). Un taux de chloration de $1,5 \mathrm{mg} \mathrm{Cl} / \mathrm{L}$ en présence de $0,3 \mathrm{mg} \mathrm{N}-\mathrm{NH}_{4}+/ \mathrm{L}$ (résultats non représentés) a en outre conduit à la formation de $9,2 \mu \mathrm{g} \mathrm{CHI} / \mathrm{L}$ après $6 \mathrm{~h}$ de temps de contact. La teneur en chloramines formées ne constituerait donc pas un facteur limitant dans la production d'iodoforme.

\section{Influence de la chloramination sur la formation des composés organohalogénés}

L'eau brute $\mathrm{EB} 1(\mathrm{pH}=8,0)$ dopée par différentes concentrations d'ions iodure $(50,100$ ou $200 \mu \mathrm{g} \mathrm{I} / \mathrm{L})$ ou d'iode $\left(25,50\right.$ ou $100 \mu \mathrm{g} \mathrm{I} / \mathrm{L}$ avec $1,9 \mathrm{mg} \mathrm{I} / \mathrm{mg}$ " $\mathrm{I}_{2}$ " à $\mathrm{pH}$ 8) a été oxydée par un taux de monochloramine de $1,5 \mathrm{mg} \mathrm{Cl} / \mathrm{L}$. Les figures $5 \mathrm{a}$ et $5 \mathrm{~b}$ représentent les résultats obtenus en termes de production de iodoforme après $6 \mathrm{~h}$ de temps de contact. II convient de noter que l'ajout d'iode dans les solutions a impliqué l'introduction simultanée d'ions iodure contenus dans la solution mère d'iode (1,9 $\mathrm{mg} \mathrm{I}$ - introduit/mg " $I_{2}$ " à $\left.\mathrm{pH} 8\right)$. II a néanmoins été possible d'après les données de la figure $5 a$ de quantifier la part de iodoforme formé à partir des ions iodure lors des expériences de chloramination de la figure $5 \mathrm{~b}$.
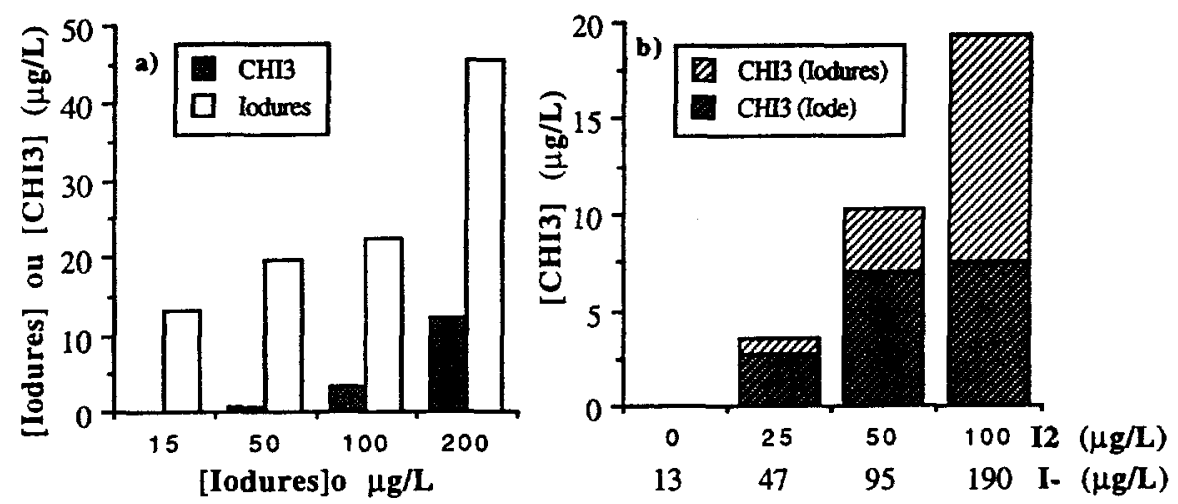

Figure 5 Chloramination de l'eau brute EB1 : formation d'iodoforme après un temps de contact de $6 \mathrm{~h}$. $\left[\mathrm{NH}_{2} \mathrm{Cl}\right]_{0}=1,5 \mathrm{mg} \mathrm{Cl} / \mathrm{L} ; \mathrm{pH}_{0}=8,0 ; \mathrm{T}=20 \pm 2{ }^{\circ} \mathrm{C}$

a) pour différentes concentrations initiales en ions iodure $\left(\left[I^{-}\right]_{\mathrm{EB} 1}=15 \mu \mathrm{g} / \mathrm{L}\right)$

b) pour différentes concentrations en iode et en ions iodure $\left(\left[I^{-}\right]_{E B 1}=\right.$ $13 \mu g /$ L)

Chloramination of the EB1 raw water : iodoform formation after 6 hours of contact time. $\left[\mathrm{NH}_{2} \mathrm{Cl}\right]_{0}=1.5 \mathrm{mg} \mathrm{Cl} / 2 ; p \mathrm{H}_{0}=8.0 ; \mathrm{T}=20 \pm 2{ }^{\circ} \mathrm{C}$

a) for different initial concentrations of iodide ions $\left(\left[\mathrm{I}_{\mathrm{EBI}}=15 \mu \mathrm{g} / \mathrm{L}\right)\right.$

b) for different concentrations of iodide ions and iodine $\left([I]_{E B 1}=13 \mu \mathrm{g} / \mathrm{L}\right)$ 
Les résultats obtenus indiquent que la production de iodoforme augmente avec la concentration en ions iodure ou en iode présent principalement sous la forme d'acide hypoiodeux en solution à $\mathrm{pH} 8,0$. La présence d'iode conduit lors de la chloramination à une production d'iodoforme plus élevée que des teneurs comparables en ions iodure. Ainsi, une concentration en "iode " de $100 \mu \mathrm{g} / \mathrm{L}$ conduit à une production d'iodoforme de $7,5 \mu \mathrm{g} / \mathrm{L}$ alors que la concentration obtenue à partir de $100 \mu \mathrm{g} / \mathrm{L}$ d'ions iodure n'est que de $3,4 \mu \mathrm{g} / \mathrm{L}$ (figure $5 \mathrm{~b}$ ).

\section{Action de l'iode}

Dans le but de déterminer si l'iode peut être l'entité active dans le mécanisme de formation du iodoforme, l'eau brute EB1 a été traitée par différentes doses d'iode ( $50 \mu \mathrm{g} / \mathrm{L}$ à $2 \mathrm{mg} / \mathrm{L}$ avec $1,9 \mathrm{mg} \mathrm{I} / \mathrm{mg}$ " $\mathrm{I}_{2}$ ") en présence d'ions ammonium et sans introduction d'un autre oxydant (ni chlore, ni chloramine). Le tableau 1 présente les teneurs en iodoforme formé après un temps de contact de $6 \mathrm{~h}$ pour différents taux d'iode à pH $8 \pm 0,2$.

Tableau 1 Oxydation par l'iode de l'eau brute EB1: Influence de la teneur en iode sur la formation d'iodoforme; $\left[\mathrm{N}-\mathrm{NH}_{4}{ }^{+}\right]_{0} \sim 0,8 \mathrm{mg} / \mathrm{L}$; Teneur en ions iodure : $1,9 \mathrm{mg} \mathrm{I} / \mathrm{mg}$ " $\mathrm{I}_{2}$ "; $\mathrm{pH}_{0} \sim 8,0$.

Table 1 Oxidation of the EBI raw water by iodine: Influence of the iodine dose on jodoform formation; $\left[\mathrm{N}-\mathrm{NH}_{4}{ }^{+}\right]_{0}-0.8 \mathrm{mg} / \mathrm{L}$; lodide ion content of solutions: $1.9 \mathrm{mg} / \mathrm{mg} \approx \mathrm{I}_{2} \approx ; \mathrm{pH}_{0} \sim 8.0$.

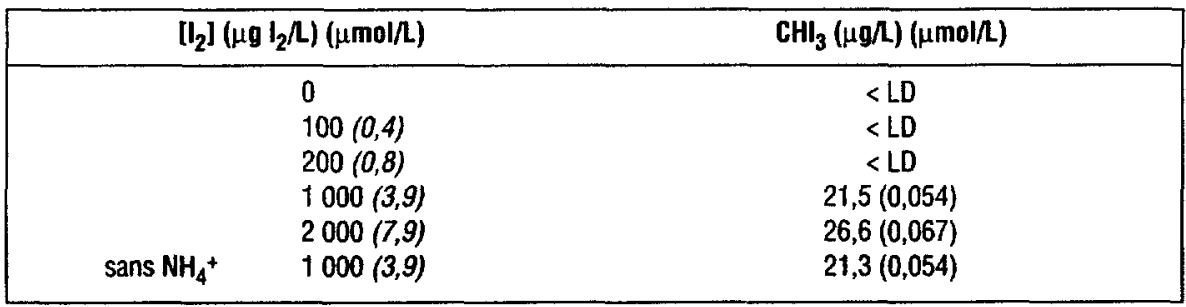

LO : Limite de détection $(0,3 \mu g / \mathrm{l})$.

On note dans ces conditions la présence d'iodoforme uniquement pour les deux concentrations les plus élevées en iode ( 1 et $2 \mathrm{mg} / \mathrm{L})$. Cette teneur semble indépendante de la présence des ions ammonium. L'association iode/ion ammonium ne représente donc pas une voie réactionnelle majoritaire dans le mécanisme de formation d'iodoforme au pH étudié. De plus ces résultats indiquent que l'iode issu de l'oxydation par le chlore des ions iodure aux concentrations rencontrées dans les eaux naturelles $\left(\left[I^{-}\right] \leq 200 \mu \mathrm{g} / L\right.$ ) ne constituera pas l'espèce réactive responsable de la formation d'iodoforme.

\section{Influence du temps de contact}

La chloramination de l'eau brute EB1 dopée en ions iodure $\left(\left[1^{-}\right]=200 \mu \mathrm{g} / \mathrm{L}\right)$ ou en iode $\left(\left[I_{2}+I^{-}\right]=200 \mu \mathrm{g} / L\right)$ ainsi que la chloration de cette eau dopée en ions iodure en présence d'ions ammonium ont été réalisées à un taux de $1,5 \mathrm{mg} \mathrm{Cl} / \mathrm{L}$. Le suivi de l'évolution de la concentration en iodoforme effectué pendant $6 \mathrm{~h}$ montre dans chaque cas une augmentation linéaire avec le temps de contact. Les pentes des droites obtenues en présence d'ions iodure uniquement ou d'iode (respectivement $3,2 \mu \mathrm{g} \cdot \mathrm{h}^{-1} \cdot \mathrm{L}^{-1}$ et $4,7 \mu \mathrm{g} \cdot \mathrm{h}^{-1} \cdot \mathrm{L}^{-1}$ ) indiquent néanmoins que la production d'iodoforme est favorisée en présence d'iode. 


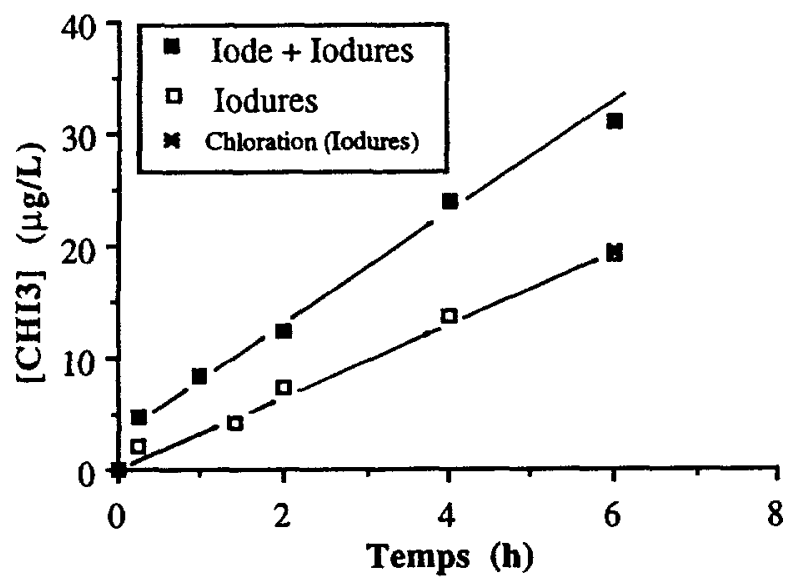

Figure 6 Chloramination de l'eau brute EB1 en présence d'iode et/ou d'ions iodure : Influence du temps de contact sur la production de iodoforme. $\left[\mathrm{NH}_{2} \mathrm{Cl}\right]_{0}$ $=1,5 \mathrm{mg} \mathrm{Cl} 2 \mathrm{~L} ;\left[\mathrm{I}^{-}\right]_{0}=200 \mu \mathrm{g} / \mathrm{L}$ ou $\left[\mathrm{I}_{2}+\mathrm{I}^{-}\right]_{0}=200 \mu \mathrm{g} / \mathrm{L} ;\left[\mathrm{N}-\mathrm{NH}_{4}{ }^{+}\right]_{0}$ $=0,6 \mathrm{mg} / \mathrm{L}$.

* Chloration (lodures) : Remplacement de la chloramination par une oxydation de l'eau brute $E B 1$ par le chlore $\left(\left[\mathrm{Cl}_{2}\right]_{0}=1,5 \mathrm{mg} / \mathrm{L}\right)$ en présence d'ions ammonium et d'ions iodure ; $\left[r_{0}=200 \mu g h\right.$.

Chloramination of the EB1 raw water with iodine and/or iodide ions: Influence of the contact time on iodoform formation. $\left[\mathrm{NH}_{2} \mathrm{Cl}_{o}=1.5 \mathrm{mg}\right.$ $\mathrm{Cl}_{2} / \mathrm{L} ;\left[\mathrm{I}_{0}=200 \mu \mathrm{g} / \mathrm{L}\right.$ or $\left[\mathrm{I}_{2}+\mathrm{r}_{0}=200 \mu \mathrm{g} / \mathrm{L} ;\left[\mathrm{N}-\mathrm{NH}_{4}{ }^{+}\right]_{0}=0.6 \mathrm{mg} / \mathrm{L}\right.$.

- Chloration (lodures): Chlorination of the $E B 1$ raw water $\left(\left[\mathrm{Cl}_{2}\right]_{0}=1.5 \mathrm{mg} \mathrm{L}\right)$ in the presence of ammonia and iodide ions; $\left[\mathrm{T}_{0}=200 \mu \mathrm{gh}\right.$.

La formation d'iodoforme obtenue à partir de la chloration en présence d'ions ammonium et d'ions iodure est équivalente à la teneur observée lors de la chloramination. La formation rapide de la monochloramine ne constitue pas une étape cinétiquement limitante dans le mécanisme conduisant au iodoforme.

\section{Chloration d'amines organiques}

À partir de l'eau brute EB1 préalablement nitrifiée, l'impact, sur la formation d'iodoforme, de deux molécules organoazotées, la glycine et la méthylamine fréquemment présentes dans les eaux naturelles, a été examiné. Pour cela, l'eau EB1 a été dopée par l'acide aminé ou l'amine à une concentration de $50 \mu \mathrm{mol} / \mathrm{L}$, équivalente à la teneur en azote ammoniacal généralement présente dans cette eau brute. Après ajout d'ions iodure $(200 \mu \mathrm{g} / \mathrm{L})$ différents taux de chloration ont été appliqués. Les figures $7 \mathrm{a}$ et $7 \mathrm{~b}$ présentent la production des THM ainsi que l'évolution des différentes formes du chlore observées après un temps de contact de $6 \mathrm{~h}$ en fonction du taux de chloration pour les eaux dopées respectivement en glycine et en méthylamine.

La courbe du point de rupture obtenue en présence de glycine est comparable à celle obtenue avec l'ion ammonium. Le point de rupture, associé au rapport molaire $\mathrm{Cl}_{2} /$ Glycine $=2 \mathrm{~mol} / \mathrm{mol}$, correspond à la destruction des chloroaminoacides formés. La chloration de l'eau en présence de méthylamine indique la persistance de chlore combiné simultanément à l'apparition de chlore libre pour les taux supérieurs à $2 \mathrm{~mol} \mathrm{Cl} / \mathrm{mol}$ Méthylamine. Le dosage des THM révèle la for- 
mation des THM chlorés et bromés en quantité croissante à partir du taux de $1 \mathrm{~mol} \mathrm{Cl} / \mathrm{mol}$ d'amine ou d'acide aminé. Pour ce qui concerne le iodoforme, ce composé est formé lors de la chloration de la glycine à des taux inférieurs à $1 \mathrm{~mol} / \mathrm{mol}$ c'est-à-dire correspondant à la présence de $\alpha \mathrm{N}$-chloroaminoacide. La concentration observée lors de la chloration de la méthylamine est plus importante (pour $\mathrm{Cl}_{2} / \mathrm{N}_{\text {organique }}=0,56 \mathrm{~mol} / \mathrm{mol},\left[\mathrm{CHI}_{3}\right]=12,1 \mu \mathrm{g} / \mathrm{L}$ à partir de la glycine contre $27,1 \mu \mathrm{g} / \mathrm{L}$ a partir de la méthylamine). La concentration en iodoforme formé en présence de méthylamine passe par un maximum pour le rapport $\mathrm{Cl}_{2}$ /Méthylamine $=1,1$ puis s'annule au-delà du point de rupture malgré l'existence de dichlorométhylamine résiduelle (PONCIN et al., 1984). La monochlorométhylamine comme la monochloramine constituerait donc un intermédiaire azoté chloré impliqué dans la formation d'iodoforme.
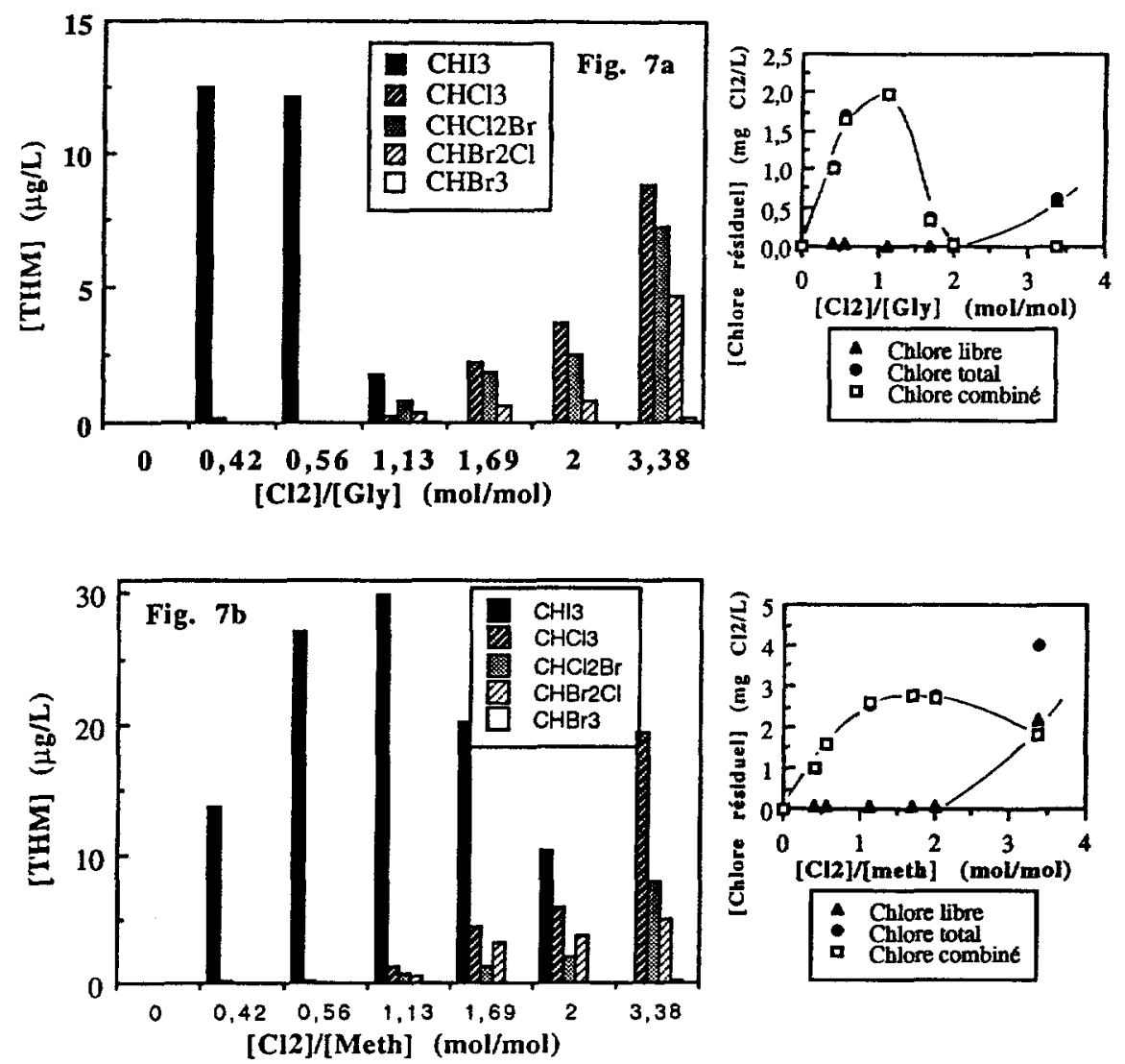

Figure 7 Influence du taux de chloration de l'eau brute EBt sur les différentes formes du chlore et sur la formation des THM. $\left[I^{-}\right]_{0}=200 \mu \mathrm{g} / \mathrm{L}$.

a) Eau dopée en glycine; $[\mathrm{Gly}]_{0}=50 \mu \mathrm{mol} / \mathrm{L}$.

b) Eau dopée en méthylamine; $[\text { Meth }]_{0}=50 \mu \mathrm{mol} / \mathrm{L}$.

Influence of the chlorine dose on chlorine speciation and on THMS formation. $[r]_{0}=200 \mu \mathrm{g} / \mathrm{L}$.

a) Water spiked with glycine; $[G l y]_{o}=50 \mu \mathrm{mol} / \mathrm{L}$.

b) Water spiked with methylamine; $[\text { Meth }]_{O}=50 \mu \mathrm{moVL}$. 


\section{4 - DISCUSSION}

L'étude de la chloration de l'eau brute EB1 dopée en ions iodure en présence d'ions ammonium, qui a mis en évidence une production d'iodoforme uniquement pour les taux inférieurs au point de rupture, nous permet d'identifier la monochloramine comme la forme active dans le mécanisme de formation de ce THM iodé. La constante de vitesse de la réaction du chlore sur les ions iodure $(k=1$ à $210^{3} \mathrm{~L} \cdot \mathrm{mol}^{-1} \cdot \mathrm{s}^{-1} ; \mathrm{pH}=7,6$ ) déterminée lors d'une expérience de cinétique compétitive, est très inférieure à la constante de vitesse de la réaction du chlore sur P'ion ammonium ( $k=5,810^{6} \mathrm{~L} \cdot \mathrm{mol}^{-1} \cdot \mathrm{s}^{-1} ;$ SAUNIER, 1976). L'action compétitive du chlore sur l'ammoniaque et les ions iodure peut conduire à un système de réactions comparable à celui obtenu avec les ions bromure. Dans un système réactionnel comprenant les entités chlore, ions iodure et ions ammonium, au regard des faibles concentrations en ions iodure, le chlore réagit principalement sur l'ammoniaque pour former les chloramines. Cette réaction ne constitue pas l'étape cinétiquement limitante dans le mécanisme de formation d'iodoforme. Les chloramines en présence d'ions iodure, voire d'iode, pourraient conduire à la formation de chloroiodamines et/ou de iodamines selon des réactions analogues à celles observées à partir de l'acide hypobromeux ou de l'ion bromure (JOHNSON et al., 1982).

$$
\begin{aligned}
& \mathrm{NH}_{2} \mathrm{Cl}+\mathrm{HOI} \rightarrow \mathrm{NHICl}+\mathrm{H}_{2} \mathrm{O} \\
& \mathrm{NHICl}+\mathrm{HOl} \rightarrow \mathrm{N}_{2} \mathrm{Cl}+\mathrm{H}_{2} \mathrm{O} \\
& 2 \mathrm{NH}_{2} \mathrm{Cl}+2 \mathrm{I}^{-} \rightarrow \mathrm{NHI}_{2}+2 \mathrm{Cl}^{-}+\mathrm{NH}_{3} \\
& \mathrm{NH}_{2} \mathrm{Cl}+\mathrm{I}^{-} \rightarrow \mathrm{NH}_{2} \mathrm{I}+\mathrm{Cl}^{-}
\end{aligned}
$$

Des précurseurs ainsi formés pourraient être à l'origine de la formation de iodoforme par action sur la matière organique selon un mécanisme comportant une réaction cinétiquement lente. Si dans les eaux naturelles l'ammoniaque contrôle quantitativement la production d'iodoforme, certains composés organoazotés (amines, acides aminés) peuvent prendre part secondairement à la production des THM iodés.

Parmi les voies réactionnelles susceptibles de conduire à la formation des composés organoiodés lors de la chloration d'eaux brutes contenant des ions iodure, les étapes du schéma de la figure 8 sont proposées pour ce qui concerne le iodoforme.

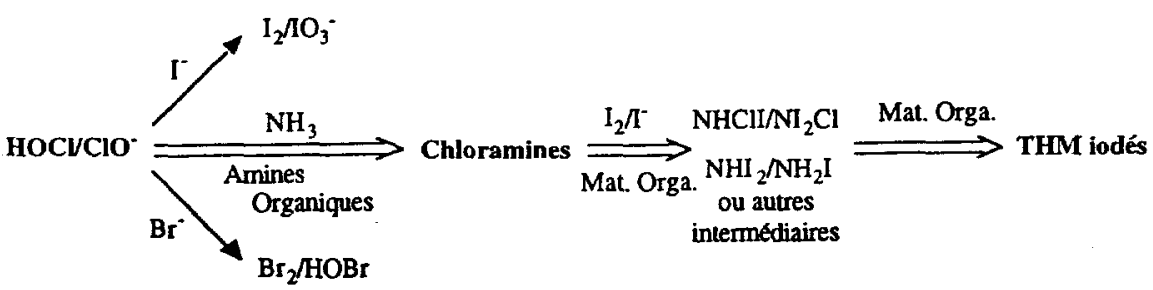

Figure 8 Formation de composés organoiodés lors de l'oxydation par le chlore d'eaux contenant des ions iodure : Schéma réactionnel.

Formation of organoiodinated compounds during chlorination of waters containing iodide ions : Proposed mechanism. 


\section{5 - CONCLUSION}

L'étude de la production d'iodoforme lors de la chloration d'une eau brute dopée en ions iodure désigne l'azote ammoniacal par l'intermédiaire de la monochloramine comme un élément essentiel au mécanisme de formation de ce THM. Les travaux réalisés parallèlement à cette étude ont ainsi montré que le dioxyde de chlore en ne réagissant pas avec les ions ammonium constitue une alternative intéressante pour inhiber la première étape du schéma réactionnel. Cependant si la présence du iodoforme permet d'expliquer le goût et l'odeur de type pharmaceutique, d'autres composés organoiodés formés lors de la chloration peuvent également prendre part à la dégradation des qualités organoleptiques de l'eau. II convient alors de définir par des études complémentaires les conditions de formation de composés organoiodés de structure plus complexe.

\section{RÉFÉRENCES BIBLIOGRAPHIQUES}

ALI-MOHAMED A.Y., JAMALI S.G.A., 1989. Potentiometric determination of fluoride and lodide in natural and processed waters of bahrain using ion-selective electrodes, Water Res., 23, 659-662.

APHA AWWA WEF, 1995. Standard Methods for the Examination of Water and Wastewater 19th Edition, Washington, DC, USA : American Public Health Association.

BRUCHET A., N'GUYEN K., MALLEVIALLE J., ANSELME C., 1989. Identification and behaviour of iodated haloform medicinal odor, Proceeding Annual Conference on Identification and Treatment of Taste and Odor Compounds, AWWA Seminar, june 18, Los Angeles, California, pp. 125-141.

GITTELMAN T.S., YOLE T.L., 1989. Treatment of iodinated haloform medicinal odors in drinking water, Proceeding Annual Conference on Identification and Treatment of Taste and Odor Compounds, AWWA Seminar, june 18, Los Angeles, California, pp. 105-122.

HANSSON R.C., HENDERSON M.J., JACK P., TAYLOR R.D., 1987. lodotorm taste complaints in chloramination, Water Res., 21(10), 1265-1271.

JOHNSON J.D., INMAN G.W., TROFE T.W., 1982. Cooling water chlorination: the kinetics of chlorine, bromide and ammonia in sea water, NTIS Ed., $121 p$.
MONTIEL A., OUVRARD J., RIGAL S., BOUSQUET G., 1987. Étude de l'origine et du mécanisme de formation de composés sapides responsables de goûts de moisi dans les eaux distribuées, T.S.M.L'EAU, 82, 73-83.

PONCIN J., LE CLOIREC C., MARTIN G., 1984. Étude cinétique de la chloration de la méthylamine par l'hypochlorite de sodium en milieu aqueux dilué, Environ. Technol. Letters, 5, 263-274.

ROLDAN-ARJONA T., GARCIA-PEDRAJAS M.D., LUQUE-ROMERO F.L., HERA C., PUEYO C., 1991. An association between mutagenicity of the Ara test of Salmonella typhimurium and carcinogenicity in rodents for 16 halogenated aliphatic hydrocarbons, Mutagenesis, 6, 199-205.

ROLDAN-ARJONA T., PUEYO C., 1993. Mutagenic and lethal effects of halogenated methanes in Ara test of Salmonella typhimurium: quantitative relationship with chemical reactivity, Mutagenesis, 8 , 127-131.

SAUNIER B., 1976. PhD Thesis, University of California at Berkeley, California.

THOMAS R.F., WEISNER M.J., BRASS H.J., 1980. The fifth trihalomethane: dichloroiodomethane, its stability and occurences in chlorinated drinking water, Water Chlorination: Environ. Impact and Health Effects, Vol. 3, Ed by Jolley R.L. et al., Ann 
Arbor Science Publishers : Ann Arbor, MI, pp. 161-168.

VENTRESQUE C., BABLON G., LEGUBE B., JADAS-HECART A., DORE M., 1990. Development of chlorine demand kinetics in a drinking water treatment plant, Water Chlorination : Chemistry, Environ. Impact and Health Effects, Vol. 6, Ed by Jolley R.L. et al., Lewis Publishers: Chelsea, MI, pp. 715-728. 